\title{
Impact of Laser Additive Enhanced Powder Feeding Methods on Melt Pool Temperature Field
}

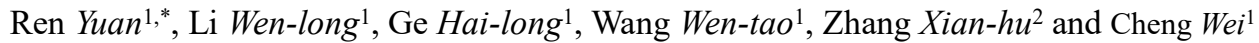 \\ ${ }^{1}$ Laser Institute of Shandong Academy of Sciences, Ji Nan, 250014, China \\ ${ }^{2}$ Remanufacturing Branch of Yan kuang Dong hua Heavy Industry Co.,Ltd, Zhou Cheng, 273500, China
}

\begin{abstract}
The surface of Q345 steel are coated with the Fe62 alloy powder by the synchronous powder feeding method and the prepositioned powder feeding method. The impacts of the two methods on the laser pool forming rate and the temperature field are tested and analyzed with a Charge-Coupled Device (CCD) colorimetric thermometer. The results indicate that the laser pool has faster forming rate, more stable and homogeneous temperature field with the synchronous powder feeding method than the prepositioned powder feeding method under the same conditions. The outcomes are of great significant to optimize the process of the laser additive enhancement technology.
\end{abstract}

Keywords: Laser Additive Enhancement; Temperature Detection; Synchronous Powder Feeding; Prepositioned Powder Feeding

\section{Introduction}

Laser additive enhancement is a new surface modification technology emerging with the development of high-power lasers in the 1970s. During the laser additive enhancement, inside the melt pool lie high energy, momentum, and mass transfer, which resulted in heat transmission, convection, and mass transfer. These directly affect the metallurgical performance and surface quality of laser for remanufacturing ${ }^{[1]}$. By detecting the distribution of the laser melt pool temperature field, we analyzed the energy, momentum, and mass transfer in the process of additive enhancement. The analysis is of great significance to the development and application of laser additive manufacturing (LAM) ${ }^{[2]}$.

Laser additive enhanced power feeding methods usually refer to synchronous powder feeding and prepositioned powder feeding. The former is to use a gas-borne powder feeder, through which laser cladding powder can be directly delivered into a spot and form a cladding layer as moving along with the spot on the workpiece surface. The latter is to place the power on the path of the laser spot in advance. With moving of the spot, the prepositioned powder together with the substrate is melted and hardened to have a laser formation layer. The two methods have direct impacts on the quality of the laser additive enhancement formation layer due to different powder melting processes ${ }^{[3]}$.

Based on the experiment that Q345 steel was used as a substrate, Fe62 as powder, and laser additive enhancement was carried out by two methods of synchronous powder feeding and prepositioned powder feeding, this paper studied the distribution of the laser melt pool temperature field and analyzed the melt pool formation and the change of the temperature field of two powder feeding methods. The experiment indicated that under the same condition, the synchronous powder feeding method could achieve faster melt pool stabilization and more uniform distribution of the temperature field as opposed to the prepositioned powder feeding. At the same time, the study results of this paper lay a theoretical and experimental foundation for the optimization of the laser additive enhancement in the application process ${ }^{[4]}$.

\section{Test Materials and Methods}

Q345 steel is used as a substrate, cladding powder is Fe62, and the powder particle size is in the range of $80-115 \mu \mathrm{m}$. A high-power optical fiber laser is used as a laser source in the experiment, the laser power is $2,000 \mathrm{~W}$, the spot diameter is $5 \mathrm{~mm}$, the focal length is $200 \mathrm{~mm}$, and the movement speed is $3 \mathrm{~mm} / \mathrm{s}$.

Before the test, we closed up the melt pool temperature field to monitor the dynamic balance state of a CCD camera and adjusted the installation angle and position and focal length of a lens. The change of the melt pool temperature field in the process of laser additive enhancement is observed via the synchronous and prepositioned powder feeding methods. Pneumatic powder feeder is adopted to feed powder and prepositioned powder feeding thickness is $3 \mathrm{~mm}$. 


\section{Experiment Results and Analysis}

\subsection{Dynamic Process and Analysis of Melt Pool Temperature Field in Laser Additive Enhancement of Synchronous Powder Feeding}

Figure 1 shows the melt pool temperature field at different moments when laser additive enhanced feeding of Fe62 metal powder. The laser is turned off at 19.82s. It can be noted from the figure that as slag and powder generated by melting Fe62 powder fall in, the heat radiation and transmission of the laser melt pool is remarkably decreased, and the heat radiation brightness at the central part of the melt pool turns dark. However, the temperature of the laser melt pool doesn't see any decrease.

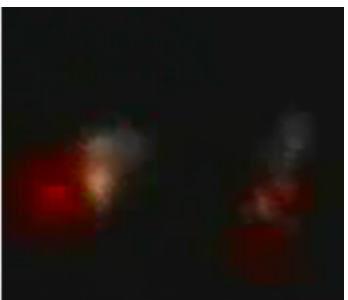

(a) $0.25 \mathrm{~s}$

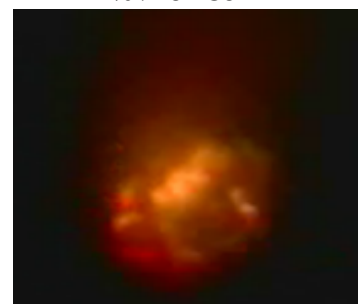

(c) $0.44 \mathrm{~s}$

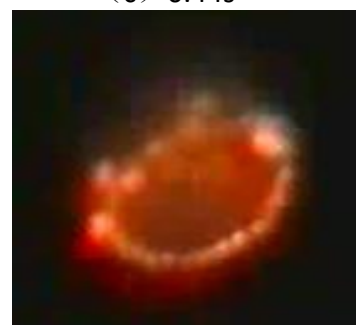

(e) $0.67 \mathrm{~s}$

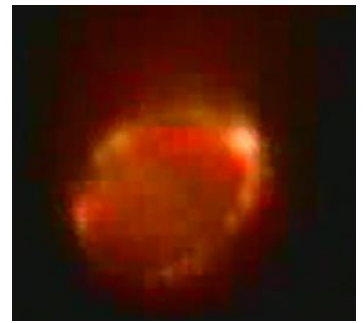

(g) $19.64 \mathrm{~s}$

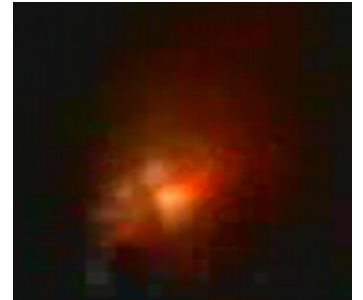

(b) $0.36 \mathrm{~s}$

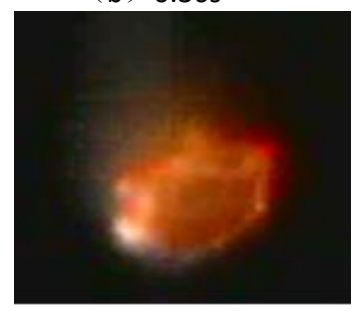

(d) $0.58 \mathrm{~s}$

(f) $18 \mathrm{~s}$

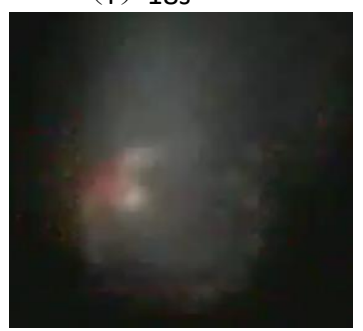

(h) $19.82 \mathrm{~s}$

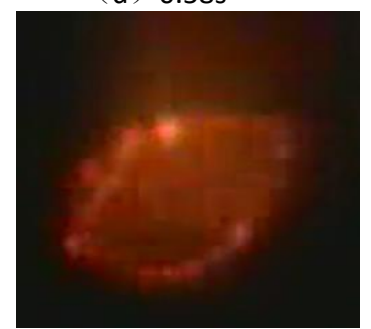

Figure 1 Heat Radiation Imaging of Lateral Powder Feeding Melt Pool

When laser radiates at $0.25 \mathrm{~s}$, a part of powder is melted. At this time, the temperature of the melting part is about $1600 \mathrm{~K}$ and heat rises at the speed of $8 \times 10^{3} \mathrm{~K} / \mathrm{s}$. As time passes, melted powder is increasing fast, and the size of the formed laser melt pool turns larger fast. At
$0.36 \mathrm{~s}$, the size is about $1.5 \mathrm{~mm}$. At $0.44 \mathrm{~s}$, it is about $2.0 \mathrm{~mm}$ while at $0.58 \mathrm{~s}$, it is increased to $3 \mathrm{~mm}$. The result shows that in the initial process, the melt pool area has extremely high heating speed. Furthermore, melting starts from the center of the laser spot, then the size of the melt pool increases rapidly. Until $0.67 \mathrm{~s}$, the melt pool enters the basically stable state. The average temperature of the melt pool remains at $1800 \mathrm{~K}$, and the size of the melt pool is about $3 \mathrm{~mm}$.

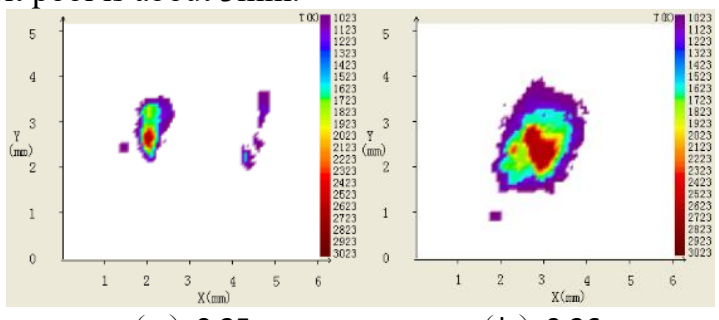

(a) $0.25 \mathrm{~s}$

(b) $0.36 \mathrm{~s}$

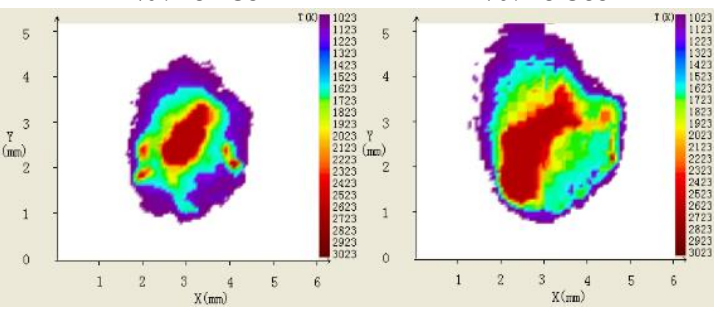

(c) $0.44 \mathrm{~s}$

(d) $0.58 \mathrm{~s}$

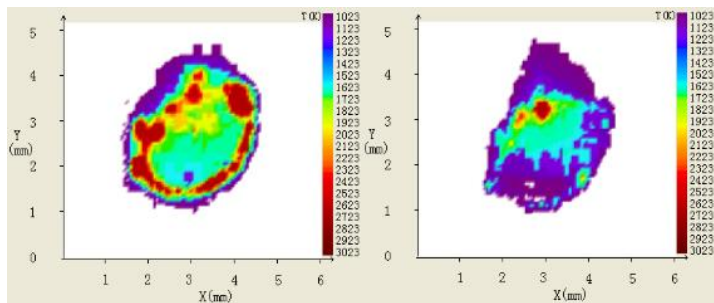

(e) $0.67 \mathrm{~s}$

(f) $18 \mathrm{~s}$

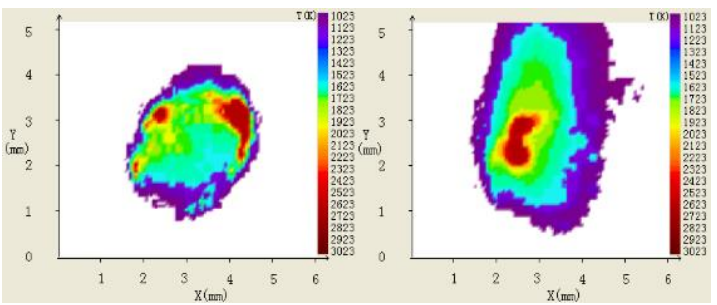

(g) $19.64 \mathrm{~s}$

(h) $19.82 \mathrm{~s}$

Figure 2 Temperature Field Distribution of Lateral Powder Feeding Melt Pool

Under the condition that powder feed, laser power, and processing speed remain constant, the temperature distribution of the melt pool is stable, and so is the size of the melt pool, which is about $3 \mathrm{~mm}$. At $19.64 \mathrm{~s}$ since the laser was turned off, the temperature of the laser melt pool goes down rapidly, the melt pool shows obvious gradient distribution of temperature. These indicated that when the laser heat input was cut off, heat transmission of the workpiece is interrupted and the temperature of the melt pool rapidly decreases. At $19.82 \mathrm{~s}$, no heat radiation images can be seen ${ }^{[5]}$. 


\subsection{Dynamic Process and Analysis of Melt Pool Temperature Field in Laser Additive Enhancement of Prepositioned Powder Feeding}

Figure 3 illustrates the heat radiation of the laser melt pool at different moments when powder is prepositioned. At $0.25 \mathrm{~s}$, the melt pool generally becomes stable and complete. At 19.82 the laser is turned off, the heat radiation of the laser melt pool is gone fast.

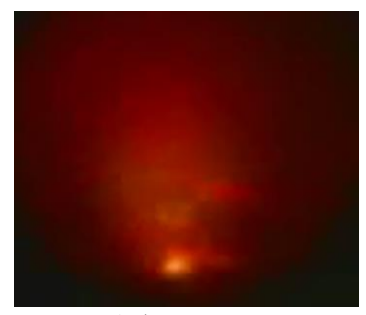

(a) $0.25 \mathrm{~s}$

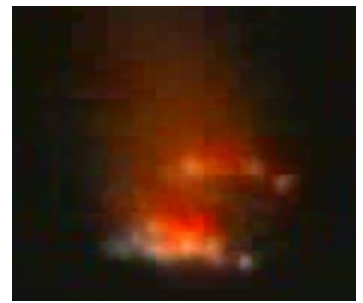

(c) $0.44 \mathrm{~s}$

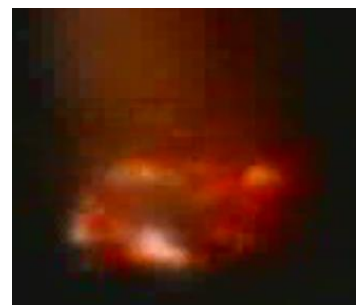

(g) $19.64 \mathrm{~s}$

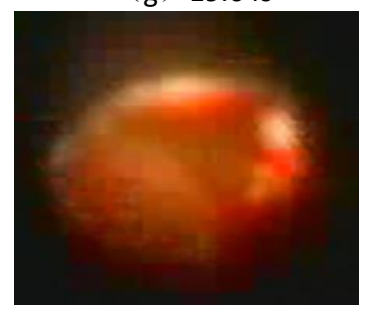

(g) $19.64 \mathrm{~s}$

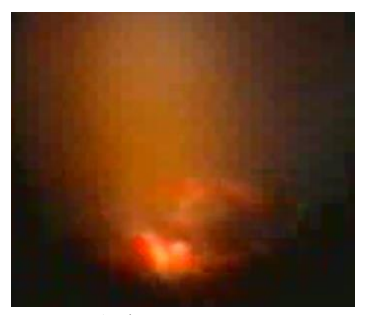

(b) $0.36 \mathrm{~s}$

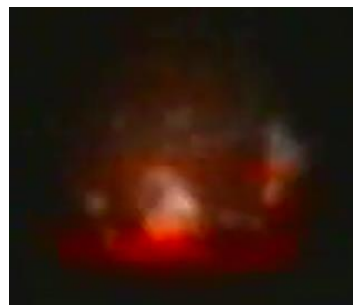

(d) $0.58 \mathrm{~s}$

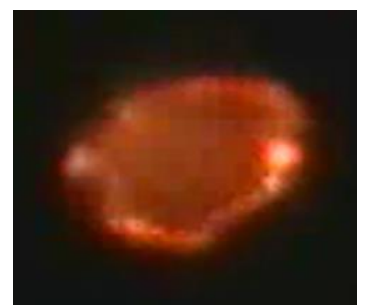

(h) $19.82 \mathrm{~s}$

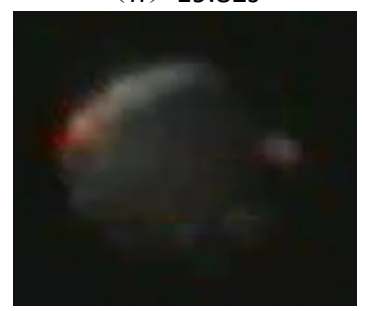

(h) $19.82 \mathrm{~s}$
Figure 3 Heat Radiation Imaging of Dynamic Process of Powder Prepositioned Laser Cladding

At the very beginning, an impurity was burned up and it can be seen that the flame is substantially symmetric to the center of the melt pool. From the beginning to $0.25 \mathrm{~s}$, only a part of powder was not melted, so that an incomplete melt pool was formed. At $0.25 \mathrm{~s}$, a complete melt pool is basically formed and starts turning stable. It can be seen from the photos of the temperature distribution between $0.67 \mathrm{~s}$ and $19.64 \mathrm{~s}$, the laser melt pool presents a stable distribution.

Figure 4 shows the temperature field distribution of the laser additive enhancement melt pool when powder is prepositioned. With the prepositioned powder feeding method, the melting point of Fe62 powder is about $1100^{\circ} \mathrm{C}$, lower than the substrate (the melting point of 45

steel is at $\left.1433^{\circ} \mathrm{C}\right)$. A laser beam firstly radiates the powder, and the powder can be melted fast to be a melt pool due to its high absorptivity ${ }^{[6]}$.

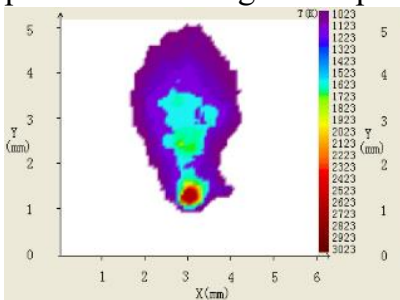

(a) $0.25 \mathrm{~s}$

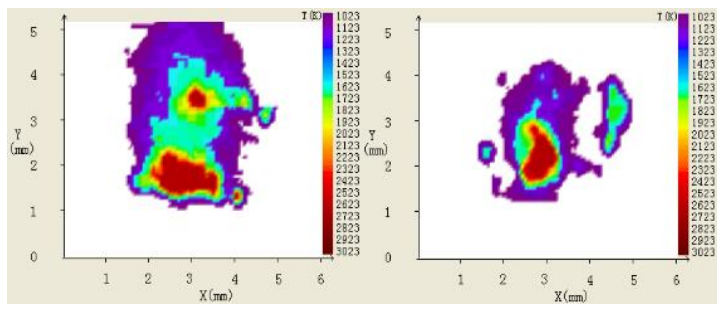

(c) $0.44 \mathrm{~s}$

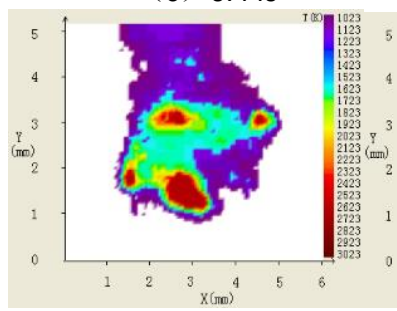

(g) $19.64 \mathrm{~s}$

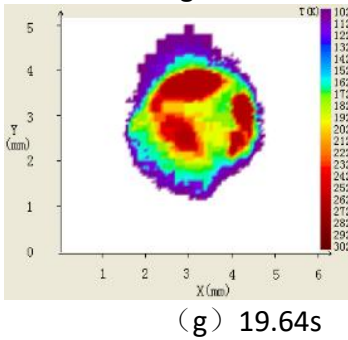

Figure 4 Temperature Field Distribution of Powder Prepositioned Laser Cladding

By comparing the changes of the melt pool temperature fields caused by the laser additive enhanced synchronous and prepositioned powder feeding methods, we found that the laser melt pool can be stable at fast and the temperature distribution more uniform under the powder feeding circumference [7]. Through analysis, during the laser additive enhancement, it can be seen that the powder of the synchronous powder feeding technology is heated uniformly, the heating area is the laser spot area, and the heat of the powder is transferred too insufficient to melt the powder. By contrast, the prepositioned powder feeding technology will transfer a part of heat to the powder around during the powder melting, and powder performance is exposed to oxygen and nitrogen in the air during heating, so the formation speed of the melt pool is slower and the formation quality is not as good as that of the synchronous powder feeding technology. However, when the melt pools formed by the prepositioned powder feeding and synchronous powder feeding methods remain stable, the 
average temperature of the melt pool formed by the former is higher than the melt pool formed by the latter. This result plays a good guidance role in the laser additive enhancement technology ${ }^{[8-10]}$.

\section{Conclusions}

1) In the process of laser additive enhancement, the quality of the formation layer can be controlled by the temperature field distribution and the size of the melt pool. It takes some time for the melt pool to be stable. According to the time and processing speed, the processing volume can be calculated in advance. In the stable state, the size of the melt pool may vary to some extent and the variation has to be taken into consideration during processing.

2) Also in this process, the average temperature before the formation of the melt pool is higher than that of the stable melt pool. This indicates that laser can generate a melt pool at instant high temperature, when the temperature is stable, so is the melt pool.

3) The analysis on the changes of the temperature fields from the synchronous and prepositioned powder feeding method of laser additive enhancement indicated that by the former method, powder is stably heated and the temperature distribution of the laser melt pool is uniform, and correspondingly the formation quality is better than that of the latter method.

\section{Acknowledgement}

This work has been supported by Major Research Project of Shan Dong Province under the project numbers 2016GGX103017 and 2016ZDJS02A09.

\section{References}

[1] Claudia H. Second Time Around and Around-Remanufacturing is Gaining Ground in Corporate America[N]. The New York Times, 1998-07-14

[2] W.Bloehs, B.Grunenwald, F.dausinger, H. Hugel. Recent progress in laser surface treatment.II. Adopted Processing for High Efficiency and Quality[J]. Journal of Laser Application, 1996, 8(1): 65 77

[3] M.Simon, T.Dowie. Disassembly process planning[C]. Proceeding of 30th International MATADOR Conference, Manchester, UK, 1993

[4] Yang Xi-chen, Wang Yun-shan, Lei Jian-bo et al.. Laser re-fabricating of expensive parts of the equipment in oil-field on the sea[C]. Lasers in Material Processing and Manufacturing II, Proceedings of SPIE(2005), 2005.1, v5629: 23 31

[5] Yang Xi-Chen, Zhao Xin, Wang Yun-shan. New Development of Laser Cladding System on Large Area for Industrial Application[C]. The Proceedings of SPIE, Nov.6-7, 1996, Vol.2888:14-20
[6] Sankaranarayanan Srikanth, Guo Wen, Kar Aravinda. Characteristics of laser-fabricated metal structures[J]. Materials and Manufacturing Processes, 1998, 13(4): 537-554

[7] R.H.Hayes. Laser powder fusion/an innovative technology for the gas turbine component repair industry[C]. World Aviation Gas Turbine Engine Overhaul and Repair'97, 1997

[8] E.Beyer. Laser macro processing -Today and tomorrow[C]. Proceedings of 4th International Congress on Laser Advanced Materials Processing-LAMP2006, May 16-19,2006, Kyoto, Japan, \#06-1

[9] Ohba Hironori, Shibata Takemasa. Temperature profiles on liquid metal surface during electron beam evaporation[J]. Japanese Journal of Applied Physics, Part 1: Regular Papers \& Short Notes \& Review Papers, 1995, 34(8A): 4253 4257

[10] Kaplinsky Michael B., Kosonocky Walter F., McCaffrey Nathaniel J., et al.. Muti-wavelength imaging pyrometer for non-contact temperature sensing[C]. Proceedings of the 1995 IEEE International Symposium on Industrial Electronics, ISIE'95. Part 1 (of 2), 1995, 1: 199 204 